\title{
Zn2+ cross-linked sodium alginate-g-allylamine-mannose polymeric carrier of rifampicin for macrophage targeting tuberculosis nanotherapy
}

\begin{abstract}
Our aim was to evaluate the capacity of polymeric nanoparticles (PNPs) to selectively deliver an antituberculosis drug (rifampicin; RF) to alveolar macrophages. Anionic biodegradable copolymer sodium alginate-g-allylamine-mannose (SA-g-AA-M) was synthesized by atom transfer free radical polymerization and direct coupling of the respective conjugates. The fabrication of RF-loaded Zn2+ ion-cross-linked SA-g-AA-M PNPs was conducted by an $\mathrm{O} / \mathrm{W}$ emulsion method followed by ionotropic gelation. The structural nature of the RFloaded SA-g-AA-M PNPs was analyzed by Fourier transform infrared (FT-IR) spectroscopy. Meanwhile, scanning electron microscopy (SEM) and transmission electron microscopy (TEM) were used to illustrate the shape and morphology of the nanoparticles. The PNPs were observed as uniform spheres in the nanometer range $(<300 \mathrm{~nm})$, with a low polydispersity index, and excellent performance in terms of drug encapsulation and release ability. The PNPs also showed strong antimicrobial activities against Mycobacterium tuberculosis. Cytotoxicity evaluation in VERO cells by an MTT assay suggested that the PNPs have good biocompatibility. Alveolar macrophage targeting was evaluated via cellular uptake by A549 cells. The cellular uptake results revealed that the $\mathrm{Zn} 2+$ concentration of the PNPs increases the intracellular concentration of RF and enhances its antitubercular efficiency. Overall, the results suggest that PNPs could lead to the development of a possible mannose-containing carrier for a macrophage-targeting drug delivery system.
\end{abstract}

Keyword: Copolymer; A549 cell; Cytotoxicity; Radical polymerization; Organic chemistry; Chemistry; Sodium 